\title{
Graphene Oxide Membranes: A Review
}

\author{
Dr I N Yadav* \\ Assistant professor, Department of chemical engineering, Bharati Vidyapeeth college of engineering Navi \\ Mumbai, 400614.
}

*Corresponding Author: Dr I N Yadav, Assistant professor, Department of chemical engineering, Bharati Vidyapeeth college of engineering Navi Mumbai, 400614.

\begin{abstract}
A perfect molecular level separating unit for any kind of species to be filtered is on very high demand. In recent years, graphene oxide has emerged as an important material which can filter ions and molecules. This is an emerging field of research which has drawn extensive attention. Membrane-based separation technology has attracted great interest in many separation fields due to its advantages of easyoperation, energy-efficiency, and easy scale-up, and environmental friendliness. The development of novel membrane materials and membrane structures is an urgent demand to promote membrane-based separation technology. Graphene oxide (GO), as an emerging star Nano-building material, has showed great potential in the membrane-based separation field. In the past few decades, membrane-based separation technology has attracted considerable attention in many separation fields due to its advantages of easy-operation, energyefficiency, and environmental friendliness.
\end{abstract}

Keywords: Membrane-based separation, Graphene oxide membranes, water purification, Nano-building material

\section{INTRODUCTION}

Since, the industrial revolution, there has been significant changes in agriculture, mining, and manufacturing sectors. Almost $71 \%$ of the Earth's surface is made up of water out of which only $2.5 \%$ is fresh water, out of which less than $1 \%$ is available for human consumption. The inaccessibility to adequate clean water is one of the most serious problems affecting large numbers of people worldwide. [1]

Numerous technologies have been employed in remediation of heavy metals in water. These technologies can be broadly classified into three processes namely: a) physio-chemical, b) biological and c) membrane processes. Among the range of appropriate technologies, membrane separation has many advantages as the chemical and energy requirements maybe less than conventional processes and treated water of high quality can be obtained.

A combination of these processes is also used to improve the treatment efficiency. So far water treatment has been mainly carried out by multiple flash distillation and reverse osmosis. Graphene is considered as an ultimate material for membrane formation because it is stronger, thinner and more chemically resistant. It is a layer of strongly packed pure carbon atoms joined together in a hexagonal honeycomb, but it is not scalable to areas required for membrane applications. There is a strong Van Der Waals force between the graphene sheets, which leads to the difficult application of graphene materials directly. Graphene oxide is the most common derivative of graphene. It has been suggested that Graphene oxide membranes, with all their favorable properties, are ideal for contaminant filtration, sieving and separation in water treatment [2]. Graphene oxide membranes continue to attract interest due to their unique molecular sieving properties combined with fast permeation rates. Graphene oxide has hydrophilicity and has been tried to use it into thin films for water treatment in recent years. [1] Membranes prepared from layers of graphene oxide offer substantial advantages over conventional materials for water treatment. However, for applications of GRAPHENE OXIDE membranes in water desalination, the Graphene Oxide interlayer spacing within the laminates has to be reduced to less than $0.7 \mathrm{~nm}$ to sieve the hydrated $\mathrm{Na}^{+}$ions from water. But due to reduction in spacing between Graphine Oxide membranes, permeation of water is significantly reduced due to reduction in hydrophilic groups, sometimes leading to formation of impermeable barrier membranes. 
On treating Graphene Oxide Membranes with HydroIodic acid; or intercalating Titania Nano sheets followed by Ultraviolet Reduction; or coating Graphene Oxide Membranes by Poly-Dopamine we can obtain Reduced Graphene Oxide Membranes. These Reduced Graphene Oxide Membranes have high permeability for water and high salt/ion rejection properties. However, PDA- Graphene Oxide membranes are environment friendly having abundant hydrophilic properties of PDA. [4]

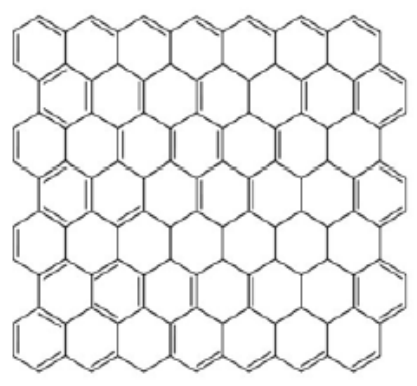

A

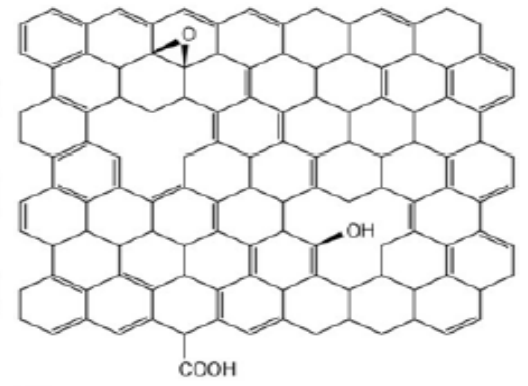

B

Fig1.1. Structure of A) Graphene B) Graphene Oxide

Graphene Oxide is hydrophilic and its surface can be easily modified with a host of biocompatible polymers such as chitosan, polyethylene glycol (PEG), poly ( $\varepsilon$-caproplactone), poly-L-lysine (PLL), and polyvinyl alcohol. These properties make it extremely attractive to a large number of scientists who apply it in the fields of drug delivery. Functionalized Graphene Oxide sheets and nanoparticles are frequently used as tissue scaffolds, fillers, and composite meshes in many areas of regenerative medicine. The membranes easily combine with host of other nanoscale materials leading to new applications in the fields of drug delivery, Tissue Engineering, cancer therapeutics, bio imaging, and diagnostics. [5] The reactive $\mathrm{COOH}$ and $\mathrm{OH}$ groups Graphene Oxide bears facilitate conjugation with various systems, such as polymers, bio molecules, quantum dots, $\mathrm{Fe}_{3} \mathrm{O}_{4}$ nano particles, etc. imparting Graphene Oxide Membranes with multi-functionalities and multi-modalities for diverse biological and medical applications. [6] Melanoma skin cancer in mice has been treated using photo thermal therapy with a near infrared laser and Graphene Oxide that was functionalized with poly- Ethylene Glycol and hyaluronic acid.[7]

\section{Preparation of Graphene Oxide}

Graphite oxide was first prepared by Oxford chemist Benjamin C. Brodie in 1859, by treating graphite with a mixture of potassium chlorate and fuming nitric acid. He reported synthesis of "paper-like foils" with $0.05 \mathrm{~mm}$ thickness. [9]

In 1957 Hummers and Offeman developed a safer, quicker, and more efficient process called Hummers' method, using a mixture of sulfuric acid $\mathrm{H}_{2} \mathrm{SO}_{4}$, sodium nitrate $\mathrm{NaNO}_{3}$, and potassium permanganate $\mathrm{KMnO}_{4}$, which is still widely used, often with some modifications. Graphene oxide has also been prepared by using a "bottom-up" synthesis method (Tang-Lau method) in which the sole source is glucose, the process is safer, simpler, and more environmentally friendly compared to traditionally "top-down" method, in which strong oxidizers are involved. Another important advantage of Tang-Lau method is control of thickness, ranging from monolayer to multi layers by adjusting growth parameters. [10]

\subsection{Synthesis of Graphene Oxide: Top Down Approach}

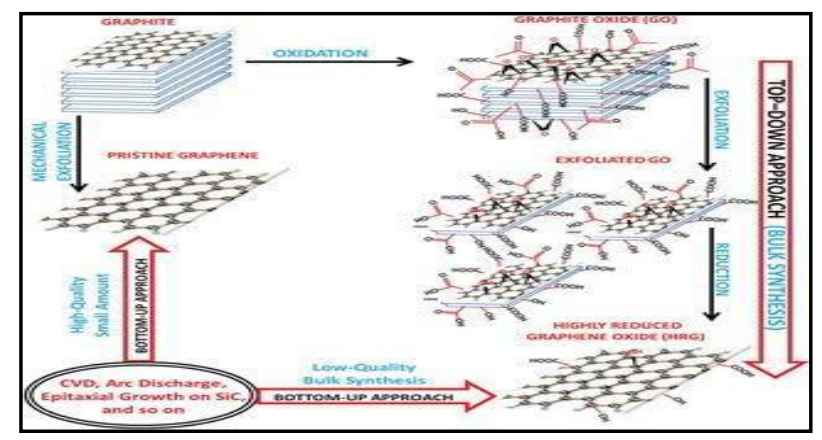

Fig1. Top -Down Approach for Synthesis of Graphene Oxide approach 
- The main drawbacks of this top-down approach are the use of strong oxidizing agents and small lateral size of Graphene Oxide Nano sheets.

- The Graphene Oxide Nano Sheets are prepared by a hydrothermal method using glucose as a sole reagent. The method is environmentally friendly, facile, low-cost as well as capable of scaling up for mass production.

\subsection{Synthesis of Graphene Oxide: Bottom Up Approach}

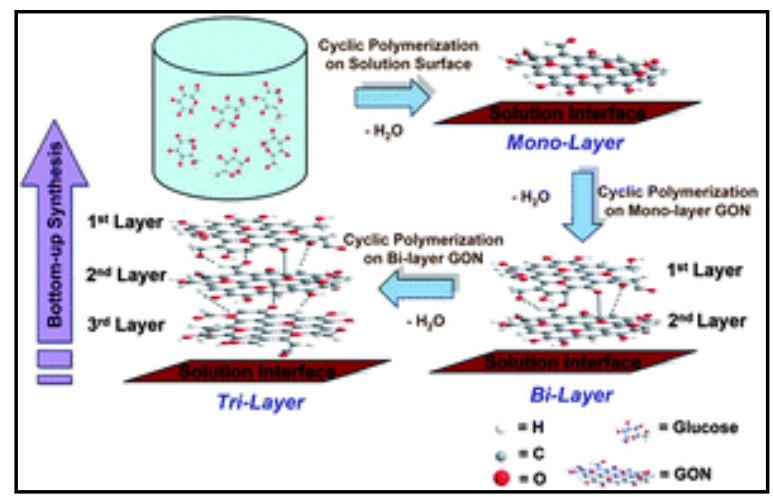

Fig2. Bottom down approach for synthesis of Graphene oxide sheets

\subsection{Various Materials Suitable for Graphene Oxide Membranes}

Normally, membrane material is manufactured from a synthetic polymer, although other forms, including ceramic and metallic "membranes," may be available. Almost all membranes manufactured for drinking water are made of polymeric material, since they are significantly less expensive than membranes constructed of other materials. Membranes constructed of polymers that react with oxidants used in drinking water treatment should not be used with chlorinated feed water. Mechanical strength is another consideration, since a membrane with greater strength can withstand larger transmembrane pressure (TMP) levels, allowing for greater operational flexibility and the use of higher pressures. Membranes with bi-directional strength may allow cleaning operations or integrity testing to be performed from either feed or filtrate side of the membrane. Membranes with a particular surface charge may remove particulate or microbial contaminants of the opposite charge due to electrostatic attraction. Membranes can also be hydrophilic (water attracting) or hydrophobic (water repelling). [13]

\section{Characterization of Graphene Oxide Membranes}

The physical chemical characterization of graphene oxide is not often of easy interpretation because this material is made of carbons and oxygen as the majority of organic molecules. Thus, the peculiar spectroscopic signals are blended by conventional carbon and oxygen signals.

\subsection{FTIR}

The FTIR is an efficient tool for a rapid characterization of Graphene Oxide. It becomes common to interpret FTIR signals as referred to hydroxyl $(\mathrm{OH})$, epoxy $(\mathrm{C}-\mathrm{O}-\mathrm{C})$ and ketone $(\mathrm{C}=\mathrm{O})$ groups. A typical FTIR spectrum is reported as below (fig3).

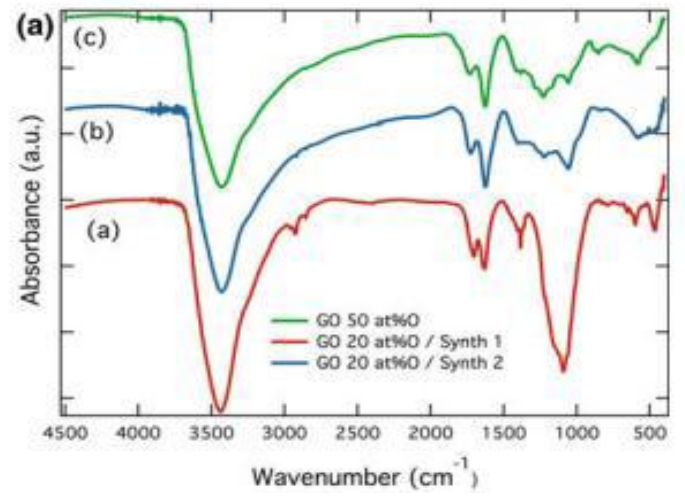

Fig3. FITR Spectra of Graphene Oxide Membrane 


\subsection{Raman Spectrum}

Raman spectrums for the GO shows only two broaden peaks which represent the G and D band. (Fig4)

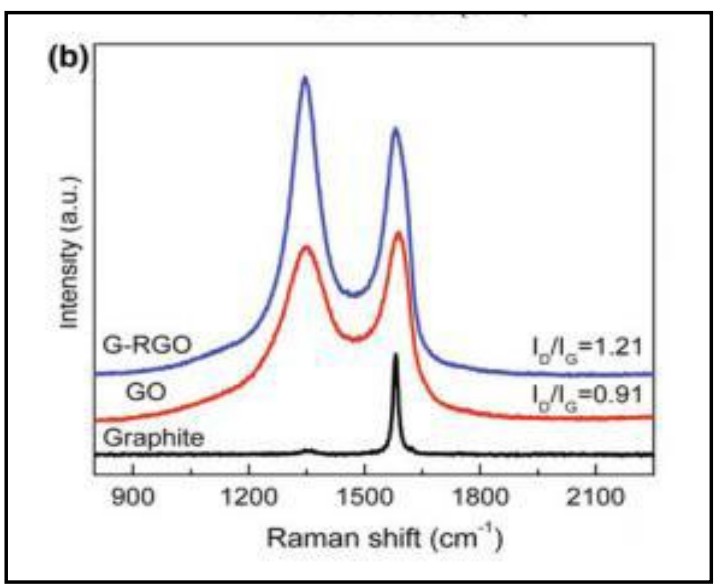

Fig4. Raman Spectrum for Graphene Oxide Membranes

\subsection{XRD} $11^{\circ}$ (Fig5).

X-ray diffraction (XRD) pattern of pristine graphene oxide shows only a broad peak around

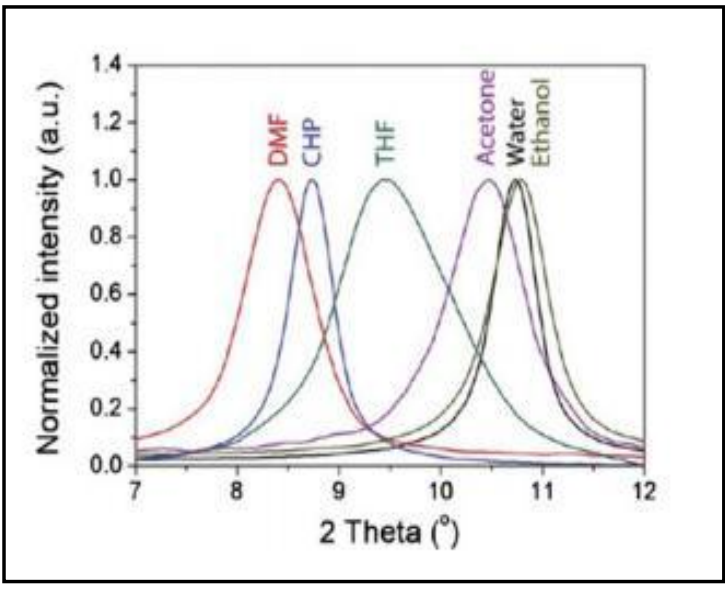

Fig5. XRD for Graphene Oxide Membranes

Various Methods for Characterization are discussed below in Table1.

Table1. Method of characterization of GO membranes

\begin{tabular}{|c|c|c|}
\hline Characterization Method & Characterization Information & Reference \\
\hline Stress-strain curves & $\begin{array}{c}\text { Mechanical stability of the membrane, tensile strength, Young's } \\
\text { modulus }\end{array}$ & 17 \\
\hline SEM & Surface morphology and cross-section structure & 18 \\
\hline AFM & Surface roughness of membrane, membrane uniformity & 18 \\
\hline CA & Surface hydrophilic or hydrophobic property of membrane & 19 \\
\hline FTIR & $\begin{array}{c}\text { Chemical structure of membrane, surface functional groups of } \\
\text { membrane }\end{array}$ & 20 \\
\hline XRD & Crystalline structures, d-spacing of membrane & 20 \\
\hline Raman Spectroscopy & Identified the existence of GO in composite membrane & 22 \\
\hline
\end{tabular}

\section{TyPes of Graphene OXIDE MEMbranes}

There are 3 types of GO membranes:

- Free-standing GO membrane

- Supported-GO membrane

- GO-modified composite membrane 


\subsection{Free-Standing}

At present, a variety of approaches have been employed to fabricate free-standing Graphene Oxide membrane, such as vacuum filtration, evaporation-driven self-assembly, self-assembly process under ambient conditions, drop casting, and pressurized ultra filtration (UF) method. Sun et al. developed free-standing Graphene Oxide membranes via a drop-casting method and investigated their water purification performance. [23]

\subsection{Supported-GO Membranes:}

Although free-standing Graphene Oxide membranes have achieved great progresses in membrane separation applications, a Graphene Oxide membrane supported on desired mechanical support for high-pressure application is rather necessary. Additionally, depositing Graphene Oxide layers onto certain polymeric or inorganic membrane surface could also improve the separation performance and antifouling property of pristine membranes.

\subsection{GO-Modified Composite Membranes:}

Apart from the two types of GO-based membrane described above, in which Graphene Oxide nano sheets were directly used as the active separation layers, researchers also focused on modifying polymeric membranes with Graphene Oxide nano sheets via different methods. By now, various Graphene Oxide-modified polymeric composite membranes have been developed and presented improved water permeability, selectivity, and anti-microbial performances.

Table2. Application of GO-based membranes.

\begin{tabular}{|c|c|c|}
\hline $\begin{array}{c}\text { Types of Graphene } \\
\text { Oxide Membrane }\end{array}$ & Name of Graphene Oxide Membrane & Application \\
\hline \multirow{4}{*}{ Free-standing } & Cross-linked GO membrane & Ion dialysis separation \\
\cline { 2 - 3 } & GOP nano hybrid membrane & Oil/water separation \\
\cline { 2 - 3 } & GO membrane & Ion penetration \\
\cline { 2 - 3 } & GO membrane & Dehydration of 85 wt \% ethanol \\
\cline { 2 - 3 } & GO/PES & Gas separation \\
\cline { 2 - 3 } & GOF/Al2O3 & Water purification \\
\cline { 2 - 3 } & GO/PAN & Water treatment \\
\cline { 2 - 3 } & GO/Nylon & Humic acid removal \\
\cline { 2 - 3 } & GO/PES & Pervaporation separation of water/ethanol \\
& GO/ceramic & mixtures \\
\cline { 2 - 3 } & IRMOF-3/GO/PDA-PSF & Water purification \\
\cline { 2 - 3 } & GO/PSF & Gas separation \\
\cline { 2 - 3 } & Pebax/GO/PVDF & Solvent resistant NF \\
\cline { 2 - 3 } GO-modified & GO/PEI/DPAN & \\
\cline { 2 - 3 } & &
\end{tabular}

\section{Applications of Graphene OXIDE Membranes}

Graphene Oxide (GO) Membranes finds its application in various ways. For example, it can be used for Desalination of water, Waste Water Treatment, Gas Separation, Nano Medicines, Molecular Separation, etc.

\subsection{Nano Medicine:}

Graphene Oxide is hydrophilic and its surface can be easily modified with a host of biocompatible polymers such as chitosan, polyethylene glycol (PEG), poly ( $\varepsilon$-caproplactone), poly-L-lysine (PLL) and polyvinyl alcohol. Graphene Oxide contains a large amount of hydrophilic groups on its edge or basal planes. [5]

These properties make Graphene Oxide extremely attractive to a large swath of scientists with new applications in the fields of drug delivery, parasitology, tissue engineering (TE), antibacterial, cancer therapy, sensors imaging, and diagnostics

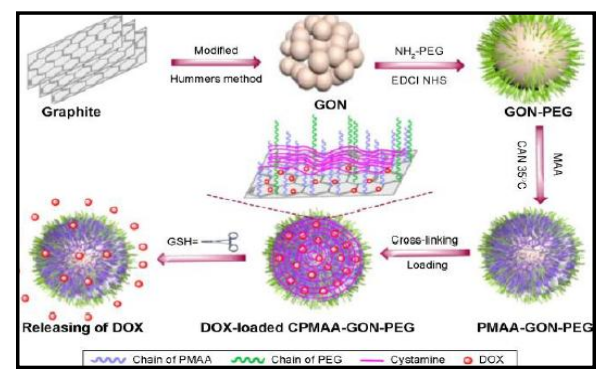

Fig6. Application Of Graphene Oxide Membrane in Drugs \& Medicines 


\subsection{Desalination of Water:}

Desalination is one of the most important and promising methods for fresh water augmentation. Permeation of water through the membrane was attributed to swelling of Graphene Oxide structures, which enables a water penetration path between individual Graphene Oxide layers. The interlayer distance of dried Hummers graphite oxide was reported as $6.35 \AA$, but in liquid water it increased to $11.6 \AA$ А. [7]

Retention rates more than $90 \%$ were reported in this study for $\mathrm{NaCl}$ solutions using stabilized Graphene Oxide membranes in a reverse osmosis setup. Graphene Oxide membranes could be used for the filtration of sea water.

\subsection{Coating Technology:}

Multilayer Graphene Oxide films are optically transparent and impermeable under dry conditions. Exposed to water (or water vapor), they allow passage of molecules smaller than a certain size. The films consist of millions of randomly stacked flakes, leaving Nano-sized capillaries between them. Graphene-coated plastic films could be used in medical packaging to improve shelf life.

\subsection{Graphene Oxide Composites and Paper-Like Materials:}

Graphene Oxide mixes readily with many polymers, forming nanocomposites, and greatly enhances the properties of the original polymer, including elastic modulus, tensile strength, electrical conductivity, and thermal stability. Free-standing Graphene Oxide films are considered for applications including hydrogen storage applications, ion conductors, and nanofiltration membranes.

\subsection{Energy Storage Device:}

Graphene Oxide has an extremely high surface area; therefore, these materials are considered for usage as electrode materials in batteries and double-layered capacitors, as well as fuel cells and solar cells.

\section{CONCLUSION}

Graphene Oxide is new emerging term in carbon nanotechnology. Graphene Oxide refers to Graphene sheet having oxygen containing groups mainly $-\mathrm{OH}$, Epoxy, carboxylic groups etc. Decorating Graphene with functional groups facilitates it to keep them detached from each other. Graphene Oxide has hydrophilic nature and can be easily dispersed in water at concentration up to $3 \mathrm{mg} / \mathrm{ml}$. Attachment of functional groups causes ${ }^{\text {ee }}$ disturbance in electronic structure yielding in poor electrical properties and reduced mechanical strength of individual Graphene sheet. On the other hand there is hope that functional groups can enhance strength of composite by good adhesion with matrix. [13]

- Good physical and mechanical properties make graphene oxide as leading material for future applications. It became subject of interest for researchers to explore and utilize its properties for various applications ranging from civil to defense and aerospace applications etc.

- High strength and modulus of graphene oxide promises its application for structural composite reinforcement

- XRD characterization confirms that along with it some trace amount of planer stacking were found even upon exfoliation

- FTIR analysis shows increase in functionality as degree of oxidation increase. Modified Hummers Method mainly attaches carboxylic groups and phenolic groups.

- In TEM analysis, it was found that graphite oxide is bunch of planes instead of single layer of graphene.

- Based on the unique single-atomic-thick and two-dimensional structure, together with excellent physicochemical property, Graphene Oxide as an emerging star Nano-building material has attracted great interest in the membrane-based separation field. 
- The separation performance of Graphene Oxide membranes could be effectively and successfully improved by different approaches, including physical approach, chemical approach, and some other novel approaches

\section{REFERENCES}

[1] Yongchen Liu,Application of graphene oxide in water treatment,College of Energy, IOP Conf. Series: Earth and Environmental Science 94 (2017) 012060

[2] Wenzheng Yu, Tong Yu and Nigel Graham, IOP publishing Development of a stable cation modified graphene oxide membrane for water treatment, 2D Materials 4 (2017) 045006

[3] Victor F. Medina, C.S. Griggs; Graphene and graphene oxide membranes for water treatment February 2016

[4] Junxian Pei, Xiantao Zhang, Lu Huang, Haifeng Jiang and Xuejiao Hu; Fabrication of reduced graphene oxide membranes for highly efficient water desalination;Royal Society of chemistry, 2016, 6, 101948

[5] Si-Ying Wu, Seong Soo A An, and John Hulme, Current applications of graphene oxide in nanomedicine, International Journal of Nanomedicine. 2015; 10: 9-24

[6] He Shen, Liming Zhang, Min Liu, and Zhijun Zhang,Biomedical Applications of Graphene, Theranostics, 2012; 2(3):283-294

[7] Sekhar C Ray, Application and Uses of Graphene Oxide and Reduced Graphene Oxide, December 2015

[8] Subhashree Priyadarsini1 Swaraj Mohanty Sumit Mukherjee Srirupa Basu Monalisa Mishra, Graphene and graphene oxide as nanomaterials for medicineand biology application, Journal of Nanostructure in Chemistry, 7 June 2018

[9] Hummers, W. S.; Offeman, R. E. (1958). "Preparation of Graphitic Oxide". Journal of the American Chemical Society. 80 (6): 1339

[10] Brodie, B. C. (1859). "On the Atomic Weight of Graphite". Philosophical Transactions of the Royal Society of London. 149: 249

[11] Libin Tang, Xueming Li, Rongbin Ji, Kar Seng Teng, Guoan Tai, Jing Ye, Changsong Wei and Shu Ping Lau, Bottom-up synthesis of large-scale graphene oxide Nano sheets, Journal of Materials Chemistry, 12,2012

[12] Mujeeb Khan, Muhammed Nawaz Tahir, Syed Farooq Adil, Hadayat Ullah Khan, M. Rafiq H. Siddiqui , Abdulrahman A. Al-warthan, Wolfgang Tremel, Graphene Based Metal and Metal Oxide Nanocomposites: Synthesis, Properties And Their Applications, Journal of Materials Chemistry

[13] Vishal S. Makadia, Studies On Synthesis And Characteriztion Of Graphene And Its Composites, Doctor of Philosophy In Materials Science, Sardar Patel University, April, 2011

[14] Zhang Y HL, Zhang Q, Peng J, Li J, ZhaiM, Yu ZZ (2012) Facile synthesis of well-dispersed graphene by $\gamma$-ray induced reduction of graphene oxide. Journal of Material Chemistry 22(26):13064

[15] Jalili R, Aboutalebi SH, Esrafilzadeh D, Konstantinov K, Moulton SE, Razal JM, Wallace GG (2013) Organic solvent-based graphene oxide liquid crystals: a facile route toward the next generation of selfassembled layer-by-layer multifunctional 3D architectures. ACS Nano 7(5):3981-3990

[16] Jinxi M, Dan Ping and Xin Dong; Recent Developments of Graphene Oxide-Based Membranes: A Review; Membranes Multidisciplinary Digital Publishing Institute; 7(2017), 52; 1-29

[17] Huang H., Mao Y., Ying Y., Liu Y., Sun L., Peng X. Salt concentration, pH and pressure controlled separation of small molecules through lamellar graphene oxide membranes. Chem. Commun. 2013; 49:5963-5965. doi: 10.1039/c3cc41953c

[18] Hu M., Mi B. Enabling Graphene Oxide Nanosheets as Water Separation Membranes. Environ. Sci. Technol. 2013; 47:3715-3723. doi: 10.1021/es400571g.

[19] Feng B., Xu K., Huang A. Covalent synthesis of three-dimensional graphene oxide framework (GOF) membrane for seawater desalination. Desalination. 2016; 394:123-130. doi: 10.1016/j.desal.2016.04.030.

[20] Guan K., Shen J., Liu G., Zhao J., Zhou H., Jin W. Spray-evaporation assembled graphene oxide membranes for selective hydrogen transport. Sep. Purif. Technol. 2017; 174:126-135. doi: 10.1016/j.seppur.2016.10.012

[21] Zheng S., Tu Q., Urban J.J., Li S., Mi B. Swelling of graphene oxide membranes in aqueous solution: characterization of interlayer spacing and insight into water transport mechanisms. ACS Nano. 2017; 11:6440-6450. doi: 10.1021/acsnano.7b02999. 
[22] Huang K., Liu G., Lou Y., Dong Z., Shen J., Jin W. A Graphene oxide membrane with highly selective molecular separation of aqueous organic solution. Angew. Chem. Int. Ed. 2014; 53:6929-6932. doi: 10.1002/anie.201401061

[23] Jia Z., Wang Y., Shi W., Wang J. Diamines cross-linked graphene oxide free-standing membranes for ion dialysis separation. J. Membr. Sci. 2016;520:139-144. doi: 10.1016/j.memsci.2016.07.042

Citation: Dr I N Yadav., (2019). “Graphene Oxide Membranes: A Review”. International Journal of Advanced Research in Chemical Science (IJARCS), 6(3), pp.25-32. DOI: http://dx.doi.org/ 10.20431/ 23490403.0603004.

Copyright: (C) 2019 Authors. This is an open-access article distributed under the terms of the Creative Commons Attribution License, which permits unrestricted use, distribution, and reproduction in any medium, provided the original author and source are credited. 